Article

\title{
Analysis of Self-Concept in Adolescents before and during COVID-19 Lockdown: Differences by Gender and Sports Activity
}

\author{
Gabriel González-Valero ${ }^{1}\left(\mathbb{D}\right.$, , Félix Zurita-Ortega ${ }^{1}$ (), David Lindell-Postigo ${ }^{2}{ }^{(D)}$, \\ Javier Conde-Pipó $^{1}{ }^{\circledR}$, Wilhelm Robert Grosz ${ }^{3}(1)$ and Georgian Badicu ${ }^{3, *} * \mathbb{( D )}$ \\ 1 Department of Didactics of Musical, Plastic and Corporal Expression, University of Granada, \\ Campus de Cartuja, s/n 18071 Granada, Spain; ggvalero@ugr.es (G.G.-V.); felixzo@ugr.es (F.Z.-O.); \\ javiconde@correo.ugr.es (J.C.-P.) \\ 2 Novaschool Sunland International, Estación de Cártama-Málaga, Carretera de Cártama Estación a Pizarra, \\ s/n 29580 Cártama, Spain; dlindell@novaschool.es \\ 3 Department of Physical Education and Special Motricity, Faculty of Physical Education and Mountain \\ Sports, Transilvania University of Brasov, 500068 Brasov, Romania; wilhelm.grosz@unitbv.ro \\ * Correspondence: georgian.badicu@unitbv.ro; Tel.: +40-769-219-271
}

Received: 3 September 2020; Accepted: 19 September 2020; Published: 21 September 2020

\begin{abstract}
An appeal has been issued to the scientific community to investigate physical, mental and emotional states, and pro-social behaviours during the COVID-19 pandemic. Hence, this study aims to investigate adolescents' self-concept before and during a lockdown period in relation to gender and type/amount of physical activity or sports. The pre-lockdown sample of 366 adolescents were aged 13-17 years $(\mathrm{M}=15.51 \pm 0.65)$, of whom $192(52.5 \%)$ were females and $174(47.5 \%)$ were males. During the lockdown, the age range of the sample was $13-17$ years $(\mathrm{M}=14.57 \pm 1.47)$, of whom $82(60.3 \%)$ were females, and 54 (39.7\%) were males. The Form-5 Self-concept Questionnaire (AF-5) was used to measure adolescents' self-concept. There was a reduction in adolescents' overall self-concept during the COVID-19 pandemic, which was positively associated with emotional well-being, with family and peers being essential factors in the development of an appropriate self-concept. Furthermore, girls' self-concept, especially academic self-concept, was higher than that of boys during the lockdown. However, both physical and emotional self-concept were higher for boys than girls before the COVID-19 lockdown, although no differences were found during the lockdown. The findings reveal that physical activity was positively correlated to self-concept before and during the COVID-19 lockdown.
\end{abstract}

Keywords: self-concept; physical activities; lockdown; COVID-19; adolescents

\section{Introduction}

Every country negatively affected by a disaster or a pandemic considers adolescents, the elderly and disabled people to be the main at-risk population groups [1-3]. The coronavirus SARS-CoV-2 infection, which leads to the disease called COVID-19 [4], caused an international state of health emergency and global pandemic [5]. This disease features respiratory infections, which directly affect the elderly $[4,6]$. However, adolescents are indirectly affected due to social distancing, educational and recreational measures adopted by the government $[7,8]$. The pandemic has had economic impacts and caused social disruptions $[9,10]$.

Furthermore, COVID-19 globally jeopardises people's mental health since it increases stress, anxiety, depression and negative social behaviour [9-11]. Therefore, adolescents are in danger of experiencing negative consequences for both mental and physical health as a result of pandemics 
and disasters [2,12]. Likewise, COVID-19 is affecting aspects of daily life, such as educational, social and leisure activities, which present both familial and emotional challenges $[7,8,13,14]$. Moreover, psychological needs, such as self-fulfilment, self-esteem and affective relationships, take on greater importance once both physiological and security requirements are met [15].

It should be noted that self-concept, which is understood as one's perception about oneself or the general opinion about self-esteem [16], may buffer people's psychological distress [17,18]. Hence, self-concept is especially important in adolescence, since everyday emotions and feelings are essential in personal development, which is subjective and changes according to external factors and new contexts of life [19]. As a matter of fact, self-concept represents a protective factor against disruptive behaviour, enhancing both mental health and positive peer relationships [20]. Consequently, the psychological construction of a positive self-concept in students during the school years produces successful socio-emotional situations and educational settings [21].

The literature has shown gender differences in well-being and self-concept. Females' well-being and self-concept are related to life satisfaction and happiness, whereas males' well-being and self-concept are related to feelings of achievement [22,23]. The effect of the COVID-19 pandemic has not been studied and no conclusive results have therefore been drawn. The need to study the association between self-concept and physical activity during this period lies in the importance of children's development, with the physical practice being a means of improving mental processes and socialisation in children [19]. Adolescents who habitually participate in physical and sports activities have a better self-concept, which is the social motor that drives better academic performance and helps their relations with peers [24].

Hence, this construct should be considered in youths and their relatives who usually experience post-traumatic symptoms under pandemic or emergency situations $[9,11,25]$, which may trigger negative mental health consequences, disruptive behaviour and a low self-concept [26-29]. As a consequence of the coronavirus pandemic, an appeal has been issued to the scientific community to pay attention to and analyse the physical, mental and emotional conditions as well as people's pro-social behaviour $[13,30,31]$. In relation to the study problem, the following research questions were suggested: (a) are there differences in adolescents' levels of self-concept before and during COVID-19? (b) are there differences between boys and girls? (c) does being physically active or inactive influence adolescents' self-concept before and during COVID-19? Therefore, this study aims to examine the level of adolescents' self-concept before lockdown and during lockdown as regards gender and physical activity, for boys and girls, and for those who are physically active and those who are not.

\section{Materials and Methods}

\subsection{Design and Participants}

This study compared the self-concept of two groups of adolescents, one pre-lockdown and one during lockdown. Convenience sampling was used to select participants, in which adolescents were asked to participate before and during the lockdown, so different samples were evaluated at two different times. As regards this selection criterion, $72.9 \%(n=366)$ of participants were assessed before the COVID-19 lockdown and 27.1\% $(n=136)$ of participants were assessed during that period. Both groups were equivalent in all respects, except for the lockdown situation. The age range of the sample before the lockdown was $13-17$ years $(M=15.51 \pm 0.65)$, while females accounted for $52.5 \%$ $(n=192)$, males accounted for $47.5 \%(n=174)$. During the lockdown, the age range of the sample was 13-17 years $(\mathrm{M}=14.57 \pm 1.47)$; females constituted $60.3 \%(n=82)$ of the total, whereas males accounted for $39.7 \%(n=54)$. In order to obtain a representative sample (error at 0.05 ; C.I. $=95 \%$ ), stratification and proportionality techniques were used when establishing the groups. 


\subsection{Instruments and Variables}

Ad-hoc questionnaire. This instrument was created by the researchers and was used to collect socio-demographic and physical and sports data. Thus, the data collected were participants' gender and age, moment (in lockdown or not), and type of physical or sport activities practised according to the adapted classification of Castro-Sánchez et al. [32]. This classification involves the following categorisation: 'None', 'Non-contact individual sports' (NCIS), 'Contact individual sports' (CIS), 'Non-contact team sports' (NCTS) and 'Contact team sports' (CTS). Furthermore, based on this classification, adolescents were classified as "physically active" and "physically inactive".

Form-5 Self-concept Questionnaire (AF-5). This questionnaire assesses one's perception about one's self-concept, which is based on the theory stated by Shavelson, Hubner and Stanton [33], and was created and validated to Spanish by García and Musitu [34]. It consists of 30 items, which use five-points Likert responses ranging from 'Never' to 'Always'. Item summation allows us to establish a general measurement of this construct, as well as to group self-concept into five dimensions: academic self-concept (A-SC; items 1, 6, 11, 16, 21 and 26), social self-concept (S-SC; items 2, 7, 12, 17, 22 and 27), emotional self-concept (E-SC; items 3, 8, 13, 18, 23 and 28), family self-concept (F-SC; items 4, 9, 14, 19, 24 and 29) and physical self-concept (P-SC; items 5, 10, 15, 20, 25 and 30). Cronbach's alpha in this study ( $\alpha=0.809)$ was similar to García and Musitu [34] research $(\alpha=0.810)$. Reliabilities of each dimension of self-concept were as follows: A-SC $(\alpha=0.853)$, S-SC $(\alpha=0.784)$, E-SC $(\alpha=0.756)$, F-SC $(\alpha=0.706)$ and P-SC $(\alpha=0.801)$.

\subsection{Procedure}

Firstly, researchers explored the range of ways to contact the population. Adolescents evaluated before the lockdown were asked thenceforth to participate in this investigation via an informative letter, which was created by the Body language department of the University of Granada and delivered through their schools. A meeting with principals was arranged afterwards, in which researchers handed over to principals some hard copies of evaluation instruments and the informative letter that needed to be delivered to adolescents' families. That letter detailed the objectives and the nature of the research, explained the voluntariness of the participation and requested informed consent. Data collection was conducted in school time during Physical Education lessons in the presence of researchers and teachers, in order to solve any doubt and to ensure a correct completion, not occurring any incidence during the process. The procedure was similar with participants evaluated during the lockdown, although protocols were done online, questionnaires were distributed via social media, and contact with families was done through schools' communication channels (e.g., email, blogs or Telegram). Anonymity was ensured in both processes, and researchers also certified that data would be used for scientific purposes. The study was conducted in full compliance with the principles expressed in the Declaration of Helsinki and was approved by the Scientific Ethical Committee of the research team's university (1230/CEIH/2020). Lastly, researchers had to invalidate 37 questionnaires due to incorrect completion.

\subsection{Data Analysis}

Descriptive analysis for variables in this study was performed, calculating mean values (M), standard deviation (SD) and frequencies (\%). Normality and homogeneity of variance for every variable were analysed through the Kolmogorov-Smirnov test. Researchers performed the independent Student's t-test to estimate differences among variables and performed the Bonferroni post-hoc test to determine the one-way variance (ANOVA) with one group, determined by Pearson's chi-squared test. Pearson bivariate correlation was calculated to establish an association among mean values, calculating a significance level of $p \leq 0.05$ and $p \leq 0.01$. Lastly, the magnitude of difference in effect size (ES) was obtained with Cohen's $d$ index [35], which is interpreted as null (0-0.19), small (0.20-0.49), medium $(0.50-0.7)$, and large $(\geq 0.80)$ [36]. Data were analysed using IBM SPSS ${ }^{\circledR}$ version 25.0 (IBM Corp, 
Armonk, NY, USA). GraphPad Prism 8 (GraphPad Prism Software Inc., San Diego, CA, USA) was used to produce figures. Lastly, 37 questionnaires were invalidated for incorrect completion, of which 26 were from individuals before the lockdown and 11 were during the COVID-19 lockdown.

\section{Results}

Table 1 presents values of self-concept regarding lockdown during the COVID-19 pandemic $(p \leq 0.05)$. The highest values of total self-concept were before the lockdown $(\mathrm{M}=3.47 \pm 0.52 ; d=0.253)$, presenting a positive association with emotional self-concept $\left(r=0.375^{* *}\right)$. This association was not present during the lockdown $(r=0.090)$. The social self-concept $(\mathrm{M}=3.70 \pm 0.75 ; d=0.401)$ was influenced by its correlation with emotional self-concept $\left(r=0.200^{* *}\right)$. The emotional self-concept $(\mathrm{M}=3.02 \pm 0.78 ; d=0.482)$ had a positive association with family self-concept $\left(r=0.143^{* *}\right)$ in both moments. Likewise, there existed a negative association between physical and emotional dimensions of self-concept during the lockdown $\left(r=-0.424^{* *}\right)$. Furthermore, while individuals evaluated before the lockdown presented higher values in family self-concept $(\mathrm{M}=4.02 \pm 0.83 ; d=0.643)$, during the lockdown presented them in the academic dimension $(\mathrm{M}=3.85 \pm 0.70 ; d=0.705)$.

Table 1. Self-concept before and duringCOVID-19 pandemic.

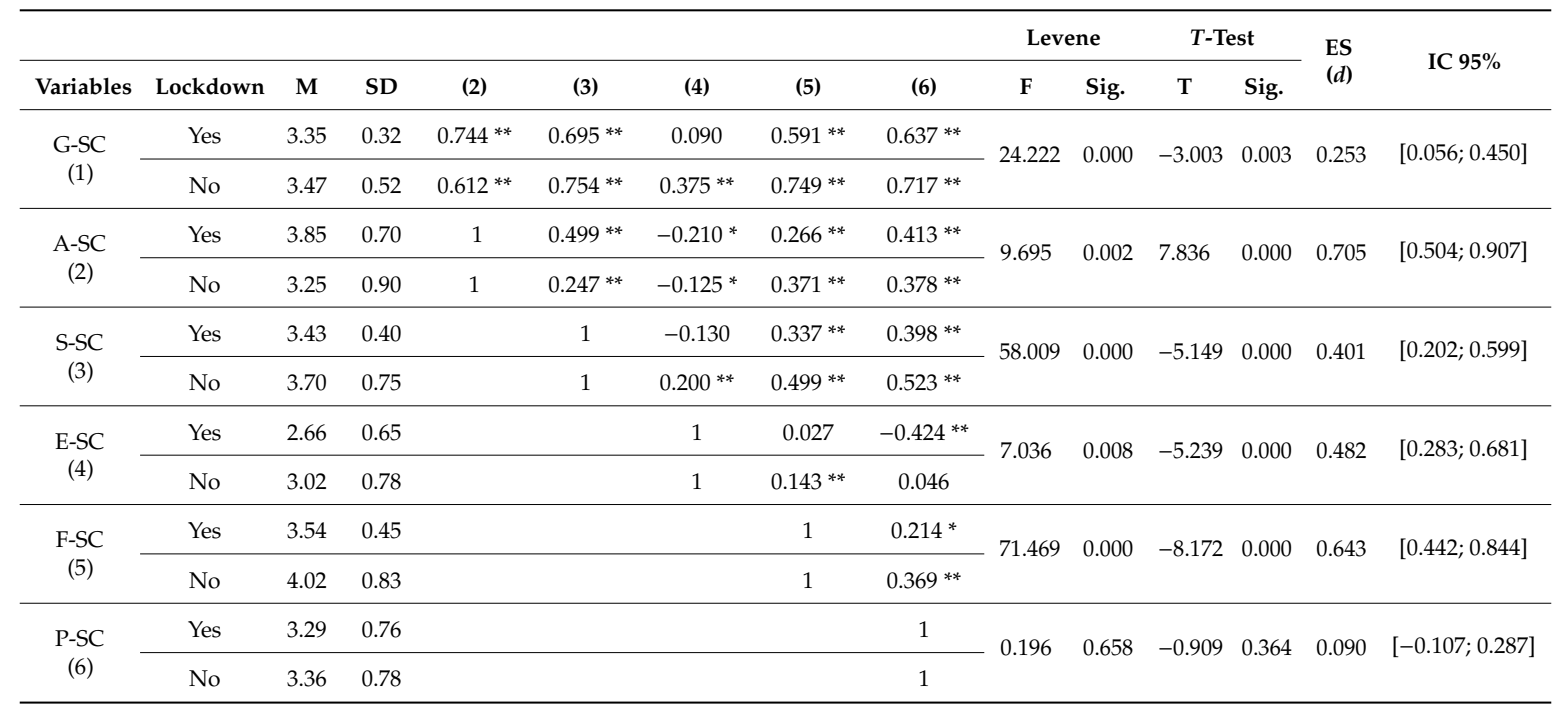

Note 1. General self-concept (G-SC); academic self-concept (A-SC); social self-concept (S-SC); emotional self-concept (E-SC); family self-concept (F-SC); physical self-concept (P-SC). Note 2. Significative correlation at $0.05(*)$; significative correlation at $0.01(* *)$.

Figure 1 shows the relation between self-conceptandthe lockdown. The general self-concept before the lockdown was higher than during this period. The same happened with the S-SC, E-SC and F-SC. In contrast, the A-SC was higher during the lockdown period. No statistically significant results were found for the P-SC.

Table 2 presents differences between gender and self-concept before the COVID-19 lockdown. Girls had a greater academic self-concept $(\mathrm{M}=3.39 \pm 0.90 ; d=0.339)$ with a highly significant difference $(p=0.001)$. However, among the boys, a negative association was shown between that dimension and emotional self-concept $\left(r=-0.287^{* *}\right)$. There was a significant difference $(p \leq 0.05)$ in some components before the lockdown. Boys had specifically higher values in the P-SC $(\mathrm{M}=3.44 \pm 0.77 ; d=0.320)$ and E-SC $(\mathrm{M}=3.14 \pm 0.73 ; d=0.298)$. However, girls' E-SC was positively correlated with the F-SC $\left(r=0.154^{*}\right)$. 

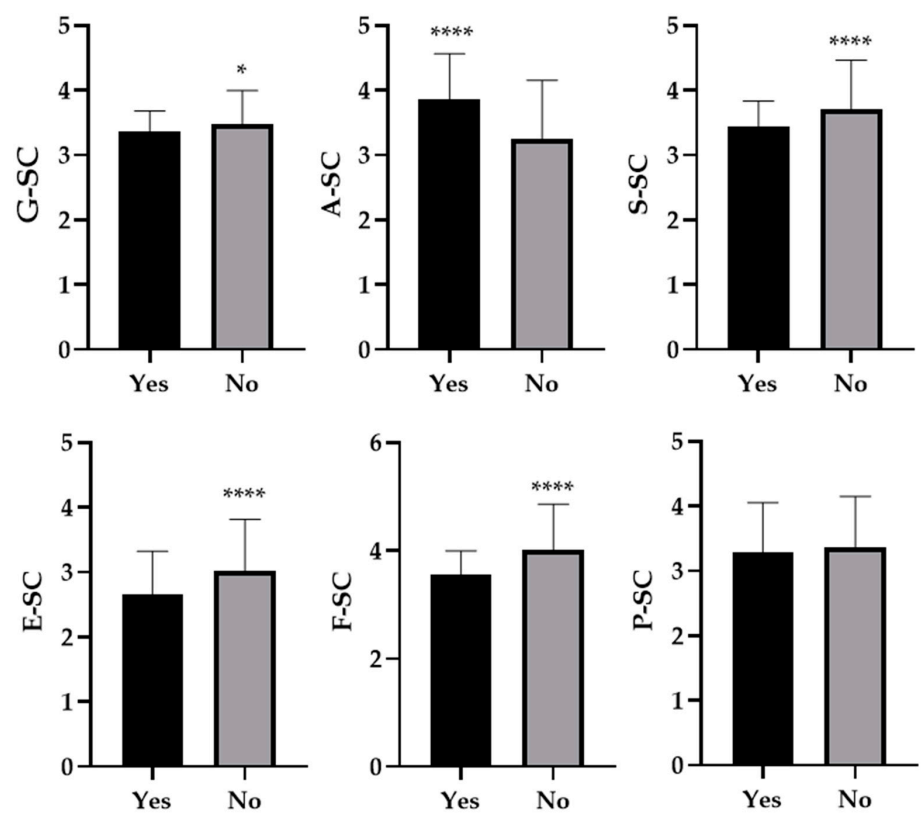

Figure 1. Self-concept and dimensions according to the period of lockdown. Note 1. Self-concept (SC); general self-concept (G-SC); academic self-concept (A-SC); social self-concept (S-SC); emotional self-concept (E-SC); family self-concept (F-SC); physical self-concept (P-SC). Note 2. Lockdown (Yes); nolockdown (No). Note 3. $p \leq 0.05\left(^{*}\right) ; p \leq 0.0001\left(^{* * * *}\right)$.

Table 2. Gender-based differences in self-concept before COVID-19 lockdown.

\begin{tabular}{|c|c|c|c|c|c|c|c|c|c|c|c|c|c|c|c|}
\hline \multirow[b]{2}{*}{ Lockdown } & \multirow[b]{2}{*}{ Variables } & \multirow[b]{2}{*}{ Sex } & \multirow[b]{2}{*}{$\mathbf{M}$} & \multirow[b]{2}{*}{ SD } & \multirow[b]{2}{*}{ (2) } & \multirow[b]{2}{*}{ (3) } & \multirow[b]{2}{*}{ (4) } & \multirow[b]{2}{*}{ (5) } & \multirow[b]{2}{*}{ (6) } & \multicolumn{2}{|c|}{ Levene } & \multicolumn{2}{|c|}{$T$-Test } & \multirow{2}{*}{$\begin{array}{l}\text { ES } \\
(d)\end{array}$} & \multirow{2}{*}{ IC $95 \%$} \\
\hline & & & & & & & & & & F & Sig. & $\mathrm{T}$ & Sig. & & \\
\hline \multirow{12}{*}{$\begin{array}{l}\text { SC before } \\
\text { lockdown }\end{array}$} & \multirow{2}{*}{$\begin{array}{c}\text { G-SC } \\
(1)\end{array}$} & B & 3.48 & 0.48 & $0.526^{* *}$ & $0.768^{* *}$ & $0.288^{* *}$ & 0.730 ** & $0.734^{* *}$ & \multirow{2}{*}{2.598} & \multirow{2}{*}{0.108} & \multirow{2}{*}{0.379} & \multirow{2}{*}{0.705} & \multirow{2}{*}{0.039} & \multirow{2}{*}[-0.167;0.244]{} \\
\hline & & G & 3.46 & 0.55 & $0.702 * *$ & $0.747^{* *}$ & $0.439 * *$ & 0.763 ** & $0.709 * *$ & & & & & & \\
\hline & \multirow{2}{*}{$\begin{array}{l}\text { A-SC } \\
(2)\end{array}$} & B & 3.09 & 0.87 & 1 & $0.165^{*}$ & $-0.287^{* *}$ & $0.220 * *$ & $0.386^{* *}$ & \multirow{2}{*}{0.026} & \multirow{2}{*}{0.873} & \multirow{2}{*}{\multicolumn{2}{|c|}{$-3.2400 .001$}} & \multirow{2}{*}{0.339} & \multirow{2}{*}[0.132;0.545]{} \\
\hline & & G & 3.39 & 0.90 & 1 & $0.342 * *$ & 0.044 & $0.493 * *$ & $0.413 * *$ & & & & & & \\
\hline & \multirow{2}{*}{$\begin{array}{l}\text { S-SC } \\
(3)\end{array}$} & B & 3.74 & 0.76 & & 1 & $0.196^{* *}$ & $0.497^{* *}$ & $0.523 * *$ & \multirow{2}{*}{0.032} & \multirow{2}{*}{0.858} & \multirow{2}{*}{0.851} & \multirow{2}{*}{0.395} & \multirow{2}{*}{0.093} & {$[-0.113 ; 0.298]$} \\
\hline & & G & 3.67 & 0.75 & & 1 & $0.197^{* *}$ & $0.505^{* *}$ & $0.519 * *$ & & & & & & \\
\hline & E-SC & B & 3.14 & 0.73 & & & 1 & 0.140 & -0.065 & 2,199 & 0.139 & 2830 & 0.005 & 0.298 & {$[0.091 ; 0.504]$} \\
\hline & (4) & G & 2.91 & 0.81 & & & 1 & $0.154^{*}$ & 0.112 & & & & & & \\
\hline & F-SC & B & 4.00 & 0.79 & & & & 1 & $0.376^{* *}$ & 3.381 & 0.067 & -0.451 & 0.652 & 0.048 & {$[-0.157 ; 0.253]$} \\
\hline & (5) & G & 4.04 & 0.88 & & & & 1 & $0.371^{* *}$ & & & & & & \\
\hline & P-SC & B & 3.44 & 0.77 & & & & & 1 & 0.024 & 0.877 & 1789 & 0.004 & 0.320 & {$[0.114 ; 0.527]$} \\
\hline & (6) & G & 3.19 & 0.79 & & & & & 1 & & & & & & \\
\hline
\end{tabular}

Note 1. Boy (B); girl(G). Note 2. Self-concept (SC); general self-concept (G-SC); academic self-concept (A-SC); social self-concept (S-SC); emotional self-concept (E-SC); family self-concept (F-SC); physical self-concept (P-SC). Note 3. Significative correlation at $0.05(*)$; significative correlation at $0.01(* *)$.

Figure 2 shows the relationship between self-concept and gender-based differences before lockdown. Girls had greater levels ofA-SC, although boys had higher E-SC and P-SC. No statistically significant results $(p \geq 0.05)$ were detected for the G-SC, S-SC and F-SC.

Table 3 presents differences according to gender regarding self-concept during the COVID-19 lockdown. There were also significant differences $(p \leq 0.05)$ during lockdown. Females had greater values in A-SC ( $\mathrm{M}=3.97 \pm 0.70 ; d=0.421)$, while for males, A-SC had a negative association with E-SC $\left(r=-0.281^{*}\right)$ and a positive correlation with F-SC $\left(r=0.414^{* *}\right)$. Significant differences were shown in G-SC $(p=0.027)$, where girls had greater values than boys $(\mathrm{M}=3.38 \pm 0.31 ; d=0.346)$. 

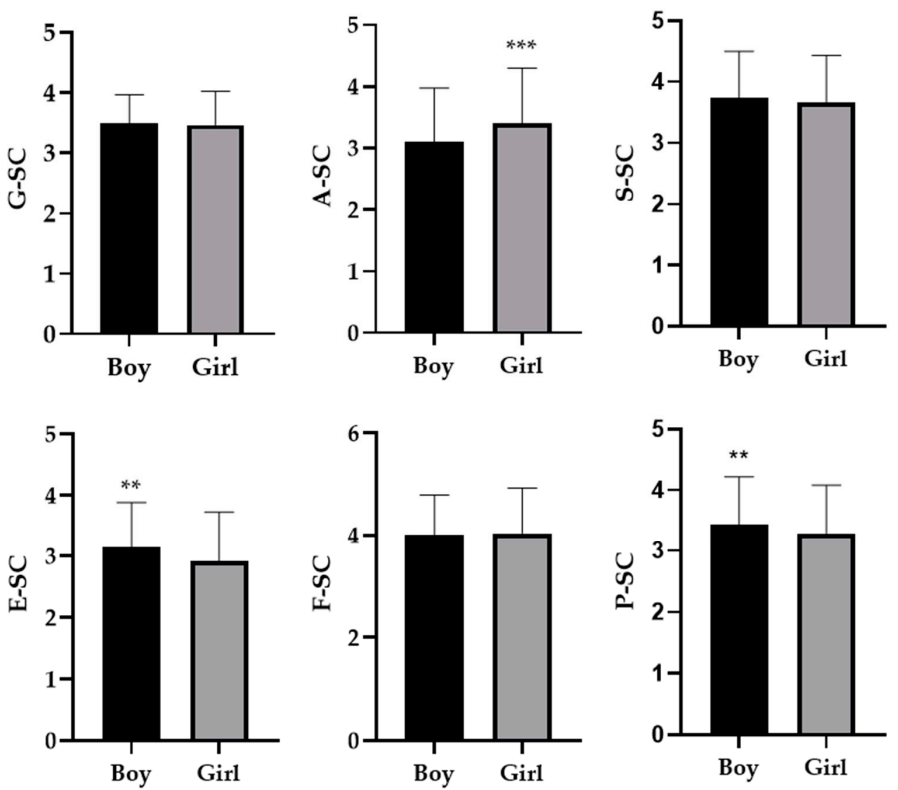

Figure 2. Gender-based differences in self-concept before COVID-19 lockdown. Note 1. Self-concept (SC); general self-concept (G-SC); academic self-concept (A-SC); social self-concept (S-SC); emotional self-concept (E-SC); family self-concept (F-SC); physical self-concept (P-SC). Note 2. $p \leq 0.01\left({ }^{* *}\right)$; $\left.p \leq 0.001{ }^{(* *}\right)$.

Table 3. Gender-based differences in self-concept during COVID-19 lockdown.

\begin{tabular}{|c|c|c|c|c|c|c|c|c|c|c|c|c|c|c|c|}
\hline \multirow[b]{2}{*}{ Lockdown } & \multirow[b]{2}{*}{ Variables } & \multirow[b]{2}{*}{ Sex } & \multirow[b]{2}{*}{$\mathbf{M}$} & \multirow[b]{2}{*}{ SD } & \multirow[b]{2}{*}{ (2) } & \multirow[b]{2}{*}{ (3) } & \multirow[b]{2}{*}{ (4) } & \multirow[b]{2}{*}{ (5) } & \multirow[b]{2}{*}{ (6) } & \multicolumn{2}{|c|}{ Levene } & \multicolumn{2}{|c|}{$T$-Test } & \multirow{2}{*}{$\begin{array}{l}\text { ES } \\
(d)\end{array}$} & \multirow{2}{*}{ IC $95 \%$} \\
\hline & & & & & & & & & & $\mathbf{F}$ & Sig. & $T$ & Sig. & & \\
\hline \multirow{7}{*}{$\begin{array}{l}\text { SC during } \\
\text { lockdown }\end{array}$} & $\begin{array}{c}\text { G-SC } \\
(1)\end{array}$ & M & 3.27 & 0.33 & $0.837^{* *}$ & 0.706 ** & -0.024 & $0.614^{* *}$ & $0.710^{* *}$ & 0.520 & 0.729 & \multicolumn{2}{|c|}{$-2.0920 .027$} & 0.346 & {$[0.001 ; 0.692]$} \\
\hline & \multirow{2}{*}{$\begin{array}{c}\text { A-SC } \\
(2)\end{array}$} & $\mathrm{M}$ & 3.68 & 0.67 & 1 & 0.596 ** & -0.281 * & $0.414^{* *}$ & $0.602 * *$ & \multirow{2}{*}{0.335} & \multirow{2}{*}{0.564} & \multirow{2}{*}{\multicolumn{2}{|c|}{$-2.3750 .019$}} & \multirow{2}{*}{0.421} & \multirow{2}{*}[0.074;0.768]{} \\
\hline & & $\mathrm{F}$ & 3.97 & 0.70 & 1 & 0.456 ** & -0.170 & 0.175 & $0.289 * *$ & & & & & & \\
\hline & $\begin{array}{l}\text { S-SC } \\
(3)\end{array}$ & $\mathrm{F}$ & 3.43 & 0.41 & & 1 & -0.072 & $0.349 * *$ & $0.339 * *$ & 0.014 & 0.905 & \multicolumn{2}{|c|}{$-0.0400 .968$} & 0.001 & {$[-0.343 ; 0.343]$} \\
\hline & \multirow{2}{*}{$\begin{array}{c}\text { E-SC } \\
(4)\end{array}$} & $\mathrm{M}$ & 2.67 & 0.63 & & & 1 & 0.009 & $-0.484 * *$ & 0.472 & 0.493 & 0.185 & 0.853 & 0.031 & {$[-0.313 ; 0.374]$} \\
\hline & & $\mathrm{F}$ & 2.65 & 0.67 & & & 1 & 0.038 & $-0.387^{* *}$ & & & & & & \\
\hline & F-SC & M & 3.54 & 0.46 & & & & 1 & 0.144 & 0.147 & 0.702 & -0109 & 0.913 & 0.001 & {$[-0.343 ; 0.343]$} \\
\hline
\end{tabular}

Note 1. Male/Boy (M); Female/Girl (F). Note 2. Self-concept (SC); general self-concept (G-SC); academic self-concept (A-SC); social self-concept (S-SC); emotional self-concept (E-SC); family self-concept (F-SC); physical self-concept (P-SC). Note 3. Significative correlation at $0.05\left(^{*}\right)$; significative correlation at $\left.0.01{ }^{* *}\right)$.

Figure 3 shows the relationships between self-concept and gender-based differences during lockdown. Especially the girls had a greater G-SC and A-SC than boys. No statistically significant results $(p \geq 0.05)$ were found for the other dimensions.

Table 4 presents the association between practising physical activities and self-concept before the lockdown $(p \leq 0.05)$. Individuals who were not involved in any physical activity had the lowest values of G-SC $(\mathrm{M}=3.30 \pm 0.49)$ compared to NCIS $(\mathrm{M}=3.58 \pm 0.52 ; d=0.554)$ and STS participants $(\mathrm{M}=3.59 \pm 0.53 ; d=0.574)$. Individuals who practiced NCIS had the highest values in A-SC $(\mathrm{M}=3.47 \pm 0.87 ; d=0.905)$ compared to individuals who did not practice any physical activity $(\mathrm{M}=2.73 \pm 0.76)$. The practice of NCIS or CTS was associated to higher values of S-SC $(\mathrm{M}=3.83 \pm 0.68$; $d=0.451$ and $\mathrm{M}=3.83 \pm 0.80 ; d=0.419), \mathrm{F}-\mathrm{SC}(\mathrm{M}=4.11 \pm 0.81 ; d=0.364$ and $\mathrm{M}=4.26 \pm 0.74 ; d=0.549)$ 
and P-SC $(\mathrm{M}=3.47 \pm 0.72 ; d=0.522$ and $\mathrm{M}=3.57 \pm 0.77 ; d=0.596)$, compared to not practising any physical activity or sport.
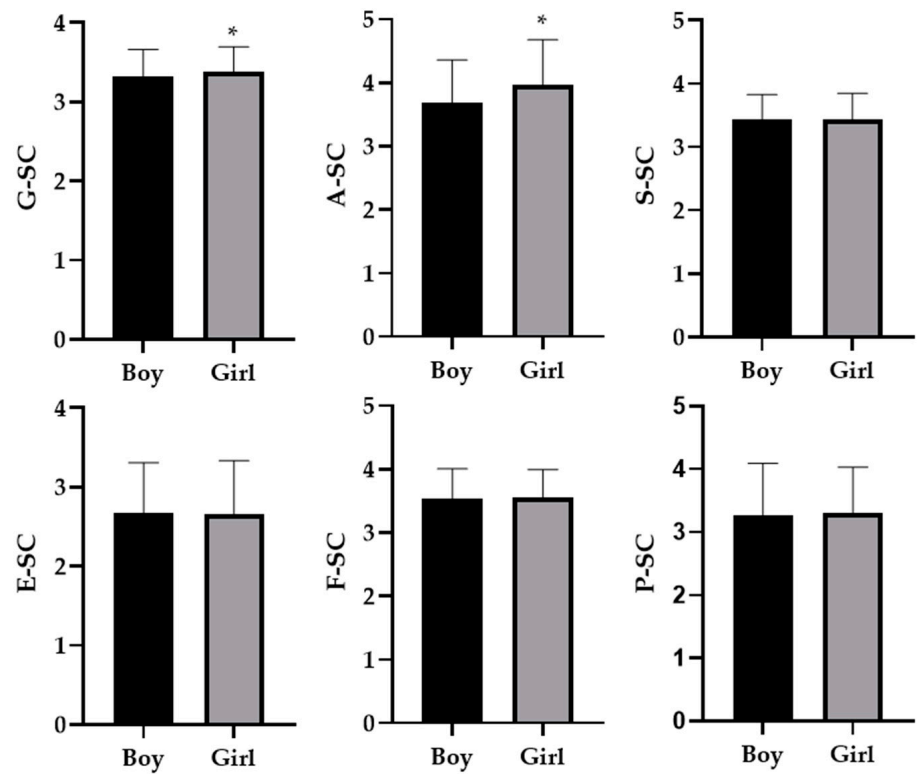

Figure 3. Gender-based differences in self-concept during COVID-19 lockdown. Note 1.Self-concept (SC); general self-concept (G-SC); academic self-concept (A-SC); social self-concept (S-SC); emotional self-concept (E-SC); family self-concept (F-SC); physical self-concept (P-SC). Note 2. $p \leq 0.05\left(^{*}\right)$.

Table 4. Practice of physical or sport activities and self-concept before the COVID-19 lockdown.

\begin{tabular}{|c|c|c|c|c|c|c|c|}
\hline Variable & Sport & Mean & SD & $\mathbf{F}$ & Sig. & $\mathrm{ES}(d)$ & IC $95 \%$ \\
\hline \multirow{5}{*}{ G-SC } & NS & 3.30 & 0.49 & \multirow{5}{*}{5.826} & \multirow{5}{*}{$p \leq 0.05^{\mathrm{a}, \mathrm{b}}$} & \multirow{5}{*}{$\begin{array}{l}0.554^{a} \\
0.574^{b}\end{array}$} & \multirow{5}{*}{$\begin{array}{l}{[0.300 ; 0.808]^{\mathrm{a}}} \\
{[0.278 ; 0.871]^{\mathrm{b}}}\end{array}$} \\
\hline & CIS & 3.35 & 0.35 & & & & \\
\hline & NCIS & 3.58 & 0.52 & & & & \\
\hline & CTS & 3.59 & 0.53 & & & & \\
\hline & NCTS & 3.46 & 0.52 & & & & \\
\hline \multirow{5}{*}{ A-SC } & NS & 2.73 & 0.76 & \multirow{5}{*}{7.492} & \multirow{5}{*}{$p \leq 0.05^{\mathrm{a}}$} & \multirow{5}{*}{$0.905^{\mathrm{a}}$} & \multirow{5}{*}[0.644;1.167]{$^{\mathrm{a}}$} \\
\hline & CIS & 3.16 & 0.88 & & & & \\
\hline & NCIS & 3.47 & 0.87 & & & & \\
\hline & CTS & 3.14 & 0.98 & & & & \\
\hline & NCTS & 3.21 & 0.74 & & & & \\
\hline \multirow{5}{*}{ S-SC } & NS & 3.50 & 0.78 & \multirow{5}{*}{3.684} & \multirow{5}{*}{$p \leq 0.05^{\mathrm{a}, \mathrm{b}}$} & \multirow{5}{*}{$\begin{array}{l}0.451^{a} \\
0.419^{b}\end{array}$} & \multirow{5}{*}{$\begin{array}{l}{[0.149 ; 0.737]^{\mathrm{a}}} \\
{[0.125 ; 0.713]^{\mathrm{b}}}\end{array}$} \\
\hline & CIS & 3.58 & 0.62 & & & & \\
\hline & NCIS & 3.83 & 0.68 & & & & \\
\hline & CTS & 3.83 & 0.80 & & & & \\
\hline & NCTS & 3.69 & 0.72 & & & & \\
\hline \multirow{5}{*}{ E-SC } & NS & 2.96 & 0.77 & \multirow{5}{*}{1.552} & \multirow{5}{*}{$p \geq 0.05$} & \multirow{5}{*}{ NP } & \multirow{5}{*}{ NP } \\
\hline & CIS & 3.31 & 0.81 & & & & \\
\hline & NCIS & 3.01 & 0.82 & & & & \\
\hline & CTS & 3.17 & 0.77 & & & & \\
\hline & NCTS & 2.88 & 0.63 & & & & \\
\hline
\end{tabular}


Table 4. Cont.

\begin{tabular}{|c|c|c|c|c|c|c|c|}
\hline Variable & Sport & Mean & SD & F & Sig. & $\mathrm{ES}(d)$ & IC 95\% \\
\hline \multirow{5}{*}{ F-SC } & NS & 3.80 & 0.89 & \multirow{5}{*}{4.210} & \multirow{5}{*}{$p \leq 0.05^{\mathrm{a}, \mathrm{b}}$} & \multirow{5}{*}{$\begin{array}{l}0.364^{a} \\
0.549^{b}\end{array}$} & \multirow{5}{*}{$\begin{array}{l}{[0.113 ; 0.615]^{\mathrm{a}}} \\
{[0.253 ; 0.845]^{\mathrm{b}}}\end{array}$} \\
\hline & CIS & 3.83 & 0.72 & & & & \\
\hline & NCIS & 4.11 & 0.81 & & & & \\
\hline & CTS & 4.26 & 0.74 & & & & \\
\hline & NCTS & 4.06 & 0.81 & & & & \\
\hline \multirow{5}{*}{ P-SC } & NS & 3.10 & 0.80 & \multirow{5}{*}{5.562} & \multirow{5}{*}{$p \leq 0.05^{\mathrm{a}, \mathrm{b}}$} & \multirow{5}{*}{$\begin{array}{l}0.522^{a} \\
0.596^{b}\end{array}$} & \multirow{5}{*}{$\begin{array}{l}{[0.299 ; 0.806]^{\mathrm{a}}} \\
{[0.299 ; 0.892]^{\mathrm{b}}}\end{array}$} \\
\hline & CIS & 3.30 & 0.51 & & & & \\
\hline & NCIS & 3.47 & 0.72 & & & & \\
\hline & CTS & 3.57 & 0.77 & & & & \\
\hline & NCTS & 3.46 & 0.86 & & & & \\
\hline
\end{tabular}

Note 1. General self-concept (G-SC); academic self-concept (A-SC); social self-concept (S-SC); emotional self-concept (E-SC); family self-concept (F-SC); physical self-concept (P-SC). Note 2. No sport(NS); non-contact individual sport (NCIS); contact individual sport (CIS); non-contact team sport (NCTS); contact team sport (CTS). Note 3. Differences between NP and NCIS $\left({ }^{\mathrm{a}}\right)$; differences between NP and CTS $\left({ }^{\mathrm{b}}\right)$.

Figure 4 compares groups of physically active and inactive individuals before the lockdown. Being physically active before lockdown was associated with higher levels of G-SC, A-SC, S-SC, F-SC and P-SC. However, no statistically significant differences $(p \geq 0.05)$ were found for the E-SC dimension.
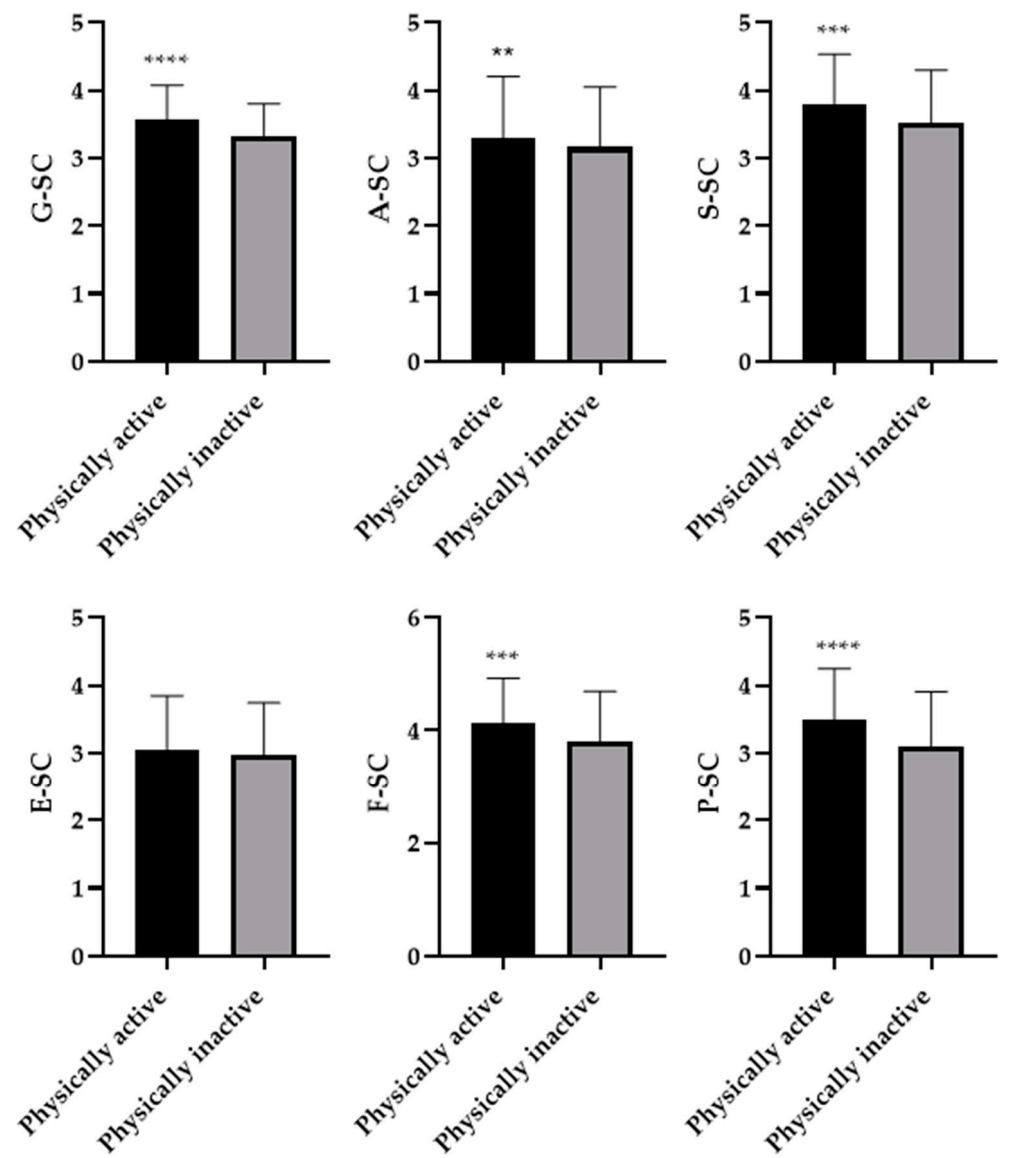

Figure 4. Self-concept and dimensions concerning physically active and inactive adolescents before the lockdown. Note 1. Self-concept (SC); general self-concept (G-SC); academic self-concept (A-SC); social self-concept (S-SC); emotional self-concept (E-SC); family self-concept (F-SC); physical self-concept (P-SC). Note 2. $\left.p \leq 0.01{ }^{* *}\right) ; p \leq 0.001\left(^{* * *}\right) ; p \leq 0.0001{ }^{(* * * *)}$. 
For the association between the practice of physical or sport activities and self-concept during the lockdown (Table 5), significant differences were found $(p \leq 0.05)$. The subjects who performed NCIS activities $(\mathrm{M}=3.48 \pm 0.26 ; d=0.827)$ were those who had higher values in G-SC compared to non-realised physical activity $(\mathrm{M}=3.22 \pm 0.66)$. No physical activity was associated with a greater A-SC $(\mathrm{M}=4.17 \pm 0.58 ; d=0.697)$. Adolescents who practiced NCIS $(\mathrm{M}=3.13 \pm 0.60 ; d=1.194)$ or CTS $(M=2.81 \pm 0.57 ; d=0.698)$ had higher values of E-SC than those not practicing any physical activity or sport $(\mathrm{M}=2.37 \pm 0.67)$. Individuals who practised NCIS $(\mathrm{M}=3.52 \pm 0.62 ; d=0.922)$ and CTS $(\mathrm{M}=3.49 \pm 0.78 ; d=0.907)$ presented the highest values of P-SC in comparison to those who did not practise any activities $(\mathrm{M}=2.88 \pm 0.76)$.

Table 5. Practice of physical or sports activities and self-concept during COVID-19 lockdown.

\begin{tabular}{|c|c|c|c|c|c|c|c|}
\hline Variable & Sport & Mean & SD & $\mathbf{F}$ & Sig. & $\mathrm{ES}(d)$ & IC 95\% \\
\hline \multirow{5}{*}{ G-SC } & NS & 3.22 & 0.36 & \multirow{5}{*}{4.209} & \multirow{5}{*}{$p \leq 0.05^{\mathrm{a}}$} & \multirow{5}{*}{$0.827^{\mathrm{a}}$} & \multirow{5}{*}[0.394;1.259]{$^{\mathrm{a}}$} \\
\hline & CIS & 3.44 & 0.12 & & & & \\
\hline & NCIS & 3.48 & 0.26 & & & & \\
\hline & CTS & 3.36 & 0.31 & & & & \\
\hline & NCTS & 3.33 & 0.28 & & & & \\
\hline \multirow{5}{*}{ A-SC } & NS & 4.17 & 0.58 & \multirow{5}{*}{3.583} & \multirow{5}{*}{$p \leq 0.05^{\mathrm{a}}$} & \multirow{5}{*}{$0.697^{a}$} & \multirow{5}{*}[0.269;1.125]{$^{\mathrm{a}}$} \\
\hline & CIS & 3.66 & 0.44 & & & & \\
\hline & NCIS & 3.64 & 0.69 & & & & \\
\hline & CTS & 3.74 & 0.67 & & & & \\
\hline & NCTS & 3.69 & 0.78 & & & & \\
\hline \multirow{5}{*}{ S-SC } & NS & 3.34 & 0.46 & \multirow{5}{*}{1.531} & \multirow{5}{*}{$p \geq 0.05$} & \multirow{5}{*}{ NP } & \multirow{5}{*}{ NP } \\
\hline & CIS & 3.38 & 0.22 & & & & \\
\hline & NCIS & 3.54 & 0.35 & & & & \\
\hline & CTS & 3.44 & 0.39 & & & & \\
\hline & NCTS & 3.35 & 0.34 & & & & \\
\hline \multirow{5}{*}{ E-SC } & NS & 2.37 & 0.67 & \multirow{5}{*}{1.125} & \multirow{5}{*}{$p \leq 0.05^{\mathrm{a}, \mathrm{b}}$} & \multirow{5}{*}{$\begin{array}{l}1.194^{a} \\
0.698^{b}\end{array}$} & \multirow{5}{*}{$\begin{array}{l}{[0.743 ; 1.645]^{\mathrm{a}}} \\
{[0.231 ; 1.164]^{\mathrm{b}}}\end{array}$} \\
\hline & CIS & 2.57 & 0.58 & & & & \\
\hline & NCIS & 3.13 & 0.60 & & & & \\
\hline & CTS & 2.81 & 0.57 & & & & \\
\hline & NCTS & 2.63 & 0.75 & & & & \\
\hline \multirow{5}{*}{ F-SC } & NS & 3.51 & 0.47 & \multirow{5}{*}{0.483} & \multirow{5}{*}{$p \geq 0.05$} & \multirow{5}{*}{ NP } & \multirow{5}{*}{ NP } \\
\hline & CIS & 3.47 & 0.55 & & & & \\
\hline & NCIS & 3.62 & 0.38 & & & & \\
\hline & CTS & 3.49 & 0.51 & & & & \\
\hline & NCTS & 3.53 & 0.37 & & & & \\
\hline \multirow{5}{*}{ P-SC } & NS & 2.88 & 0.76 & \multirow{5}{*}{5.714} & \multirow{5}{*}{$p \leq 0.05^{\mathrm{a}, \mathrm{b}}$} & \multirow{5}{*}{$\begin{array}{l}0.922^{\mathrm{a}} \\
0.907^{\mathrm{b}}\end{array}$} & \multirow{5}{*}{$\begin{array}{l}{[0.485 ; 1.359]^{\mathrm{a}}} \\
{[0.432 ; 1.382]^{\mathrm{b}}}\end{array}$} \\
\hline & CIS & 3.55 & 0.38 & & & & \\
\hline & NCIS & 3.52 & 0.62 & & & & \\
\hline & CTS & 3.49 & 0.78 & & & & \\
\hline & NCTS & 3.33 & 0.77 & & & & \\
\hline
\end{tabular}

Note 1. General self-concept (G-SC); academic self-concept (A-SC); social self-concept (S-SC); emotional self-concept (E-SC); family self-concept (F-SC); physical self-concept (P-SC). Note 2. No Sport (NS); non-contact individual sport (NCIS); contact individual sport (CIS); non-contact team sport (NCTS); contact team sport (CTS). Note 3. Differences between NP and NCIS $\left({ }^{a}\right)$; differences between NP and CTS $\left({ }^{b}\right)$. 
Figure 5 compares groups of physically active and inactive adolescents during the lockdown. Being physically active during lockdown was associated with higher levels of G-SC, A-SC, S-SC and P-SC. However, no statistically significant differences $(p \geq 0.05)$ were found in the E-SC and F-SC dimensions.
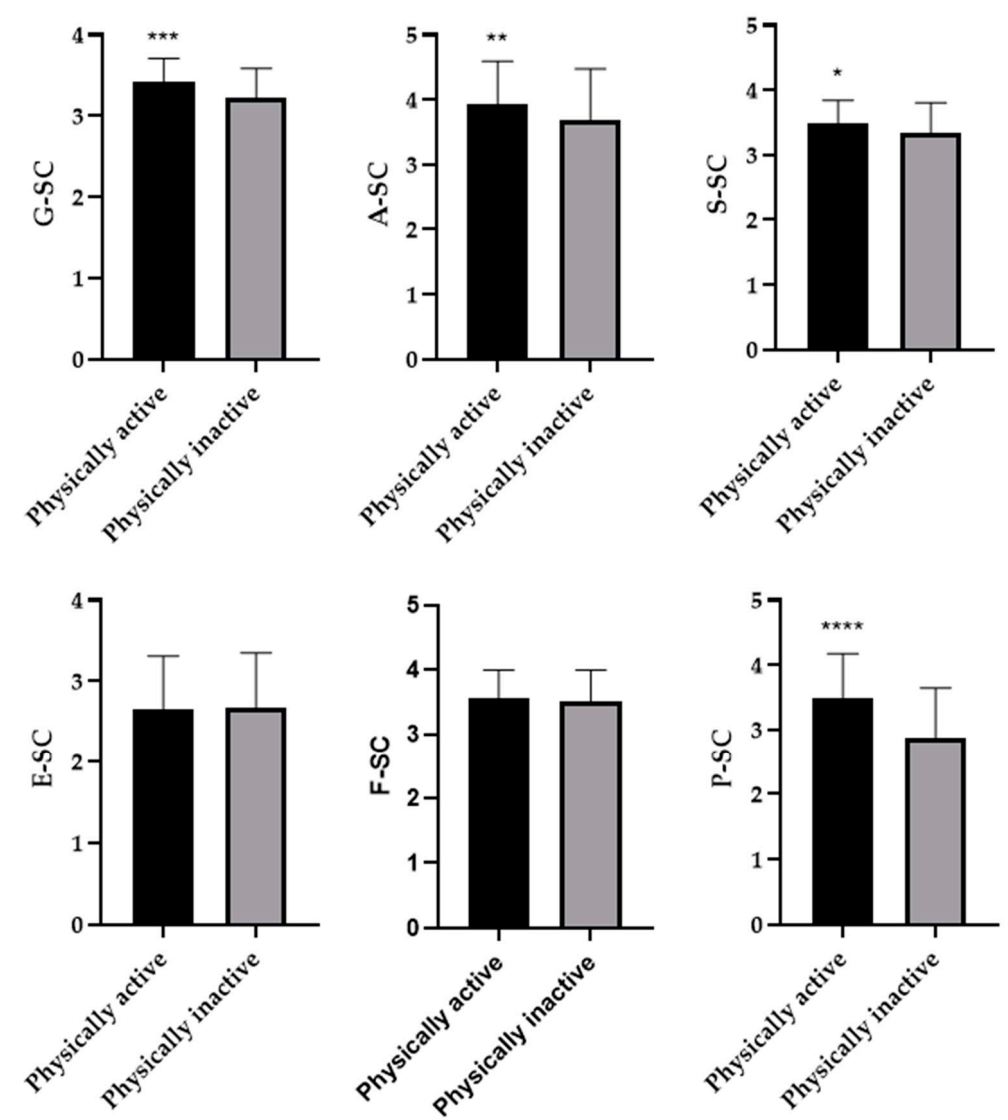

Figure 5. Self-concept and dimensions concerning physically active and inactive adolescents before the lockdown. Note 1. Self-concept (SC); general self-concept (G-SC); academic self-concept (A-SC); social self-concept (S-SC); emotional self-concept (E-SC); family self-concept (F-SC); physical self-concept (P-SC). Note 2. $\left.p \leq 0.05\left(^{*}\right) ; p \leq 0.01\left(^{* *}\right) ; p \leq 0.001\left(^{* * *}\right) ; p \leq 0.0001{ }^{* * * *}\right)$.

\section{Discussion}

This study aims to establish the association betweenself-concept, gender and the practice of physical activities, as well as to estimate the magnitude of their differences, in adolescents before and during the COVID-19 lockdown. There area wide variety of studies that addressed this field before the COVID-19 lockdown [19,37-39], yet evidence evaluating adolescents' self-concept during the COVID-19 pandemic was not found. The strength of this research relies on comparing adolescents' self-concept before and during the COVID-19 pandemic, as well as estimating differences based on gender and the practice of physical activity. This is a crucial area of research since self-concept is a pivotal construct in adolescence $[40,41]$, given its association with quality and life satisfaction, and because it is influenced by social, physical, emotional, academic and family features $[40,42,43]$.

Adolescents' general and social self-concept were higher before the lockdown. However, the emotional detriment during the lockdown caused lower values of self-concept in comparison to before the isolation. In this sense, studies such as Martínez-Marín, Martínez and Paterna [44] evinced that self-concept predicted emotional intelligence, both genders being associated with clarity and emotional repair. In line with this, an appropriate emotional state is associated with a better self-perception, self-esteem and self-rate in youth [45]. 
Emotional self-concept was higher before the lockdown, and it was correlated to contact with family and peers. Findings reveal that as adolescents' knowledge increases, so positive/negative emotion regulation and psychological adjustment are more efficient [46]. Hence, participants' family self-concept was higher before the lockdown, since adolescents who reported a high family functioning had appropriate values of self-concept [20].

However, during the COVID-19 lockdown there was a negative association between physical and emotional components, as well as higher values of academic self-concept. Staying at home, with resources enough to maintain the normality, meant that adolescents had a higher academic self-concept, which led to higher academic performance [47]. In this sense, perceived competences are demonstrated to buffer the association between academic self-concept and goal-oriented motivation [48].

As regards gender-based differences, females had higher values of academic self-concept in both moments. Moreover, males' academic self-concept was negatively correlated to emotional self-concept, yet family self-concept was positively associated with their academic self-concept during the lockdown. Furthermore, boys had higher levels in physical and emotional self-concept before the lockdown, while girls' emotional self-concept was correlated to support and proximity of family and peers. Males usually have higher emotional and physical self-concept, whereas females tend to have higher academic and family self-concept $[19,41,46,48-51]$. Age intensifies this difference, and some aspects were revealed, such as peers' relations, academic performance or motor-perceptive skills consolidation [21,52]. On the whole, girls' general self-concept was higher during the lockdown.

The gender-based difference in terms of the dimensions of self-concept causes some differences while choosing a physical activitie, non-contact individual sports and contact team sports being chosen more frequently by adolescents, which are associated with identity acceptance $[19,32,53]$. However, girls usually are less involved in these types of activity, yet benefits and enhancement on self-concept are very similar if both genders take part in physical activities in the same way [19,54]. Following on from the foregoing, practising no physical activities led to low levels of self-concept before the lockdown; indeed, there was a positive association between self-concept dimensions and the level of physical activity, and self-concept was negatively associated with sedentary behaviour [24]. In line with this, organised physical activity fostered a higher meeting of needs in the youngest [37].

Nevertheless, practising non-contact individual sports was associated with a higher academic self-concept in adolescents, as opposed to those who practised no physical activity. The practice of physical activity and the decrease in sedentary behaviour were generally correlated to academic performance and cognitive development [55-58], so that these factors are positively associated with a higher academic self-concept $[59,60]$. Likewise, adolescents who practised either non-contact individual sports or contact team sports had higher social, family and physical self-concept in comparison to those who did not practise any physical activity. Generally, it has been demonstrated that the benefits of practising a moderate physical activity in adolescence promote the development of self-concept and help to optimise health and welfare in the long term [61].

During the COVID-19 pandemic, some studies exposed that the practice of physical activity was a useful tool to cope with this situation, as well as to improve people's health and their ability to enhance their self-growth $[62,63]$. Lack of regular practice of physical activity within this period led to harmful consequences [64-70]. Nevertheless, some countries allowed the off-site practice of physical activity such as biking as a mean of transport, providing that people maintained social distance, which reported a lower health detriment due to both physical activity and social distance [71]. In this investigation, there was a positive association between the practice of individual physical activities and general/physical self-concept in adolescents. Thus, adolescents with higher emotional strength were the ones who expressed practising physical activity. In line with this, psychological consequences during COVID-19 pandemic have also been studied, so the lockdown resulted in harmful psychological consequences, although physical activity was associated with the prevention of psychology disruptions since it helps to control anxiety, decrease psychosomatic symptoms and enhance self-esteem [72-74]. However, adolescents who did not practise any physical activity during the isolation had higher values 
in academic self-concept. Time expenditure during this lockdown is focused on doing daily tasks such as assignments, practising physical activity and using IT devices [46].

The return to habitual practice of physical activity and its link with COVID-19 is not established yet. Experts are working on protocols that prevent the virus spreading so that people may practise physical or sports activities during a lockdown due to their physical, social, psychological and cognitive benefits [75-78]. This is a crucial matter because this is not the only global pandemic we are supposedly suffering in future. Lastly, it must be highlighted that the World Health Organization [79] states that an active life enhances self-perception [19].

The present study has some limitations. It is important to emphasise some caveats to the findings of this research for generalisation to other populations such as the population size. Furthermore, the descriptive design did not allow for establishing causal association (test-retest), so the results obtained ought to be interpreted cautiously. To the knowledge of the authors of this study, another limitation has been the lack of solid research that worked on the study variables during the COVID-19 pandemic. Consequently, it would be interesting to widen the sample by including more adolescents across the globe so that comparisons may be possible. Moreover, it would be interesting to evaluate the population once the COVID-19 pandemic finishes, establishing associations within the entire period. Lastly, from another perspective and as a practical application, it has been demonstrated that a cognitive-behaviour intervention and the practice of physical activities may help to obtain higher family, emotional and physical self-concept, as well as a decrease in psychopathological symptoms, in harmful situations in which psychosocial problems appear and there is a detriment of mental health $[22,30,80,81]$.

\section{Conclusions}

The aim of the study was to assess the level of self-concept of adolescents before and during the lockdown, as regards gender and physical activity. Therefore, it is concluded that adolescents' self-rate and self-concept before the lockdown were higher than during the COVID-19 pandemic, which is positively associated with emotional self-concept. Moreover, family and peers were essential factors for an appropriate emotional self-concept development before and during the lockdown. Furthermore, academic self-concept during the lockdown was higher than before it.

It may be highlighted that females' self-concept was higher during the isolation than the males' one. Specifically, girls had a higher academic self-concept in both times, although boys' academic self-concept was influenced by their emotional management and their relationships with family and peers. Even though males' physical and emotional self-concept was higher than the females' ones before the lockdown, these differences did not last during the lockdown.

As regards physical activity, people who did not practise any physical or sports activity had a lower self-concept before the lockdown. During the isolation, practising physical activity before it was associated with higher academic, social, family and physical self-concept, as opposed to those who practised no physical activity. However, academic self-concept was positively associated with not practising physical activity. Within the lockdown, adolescents who practised non-contact individual sports had higher general and emotional self-concept. Lastly, before the lockdown, adolescents who practised physical activity reported higher physical self-concept.

Author Contributions: Conceptualisation, G.G.-V. and F.Z.-O.; methodology, G.G.-V., F.Z.-O. and D.L.-P.; software, G.G.-V. and F.Z.-O.; formal analysis, G.G.-V., F.Z.-O. and J.C.-P.; investigation, G.G.-V., F.Z.-O., D.L.-P. and J.C.-P.; data curation, G.G.-V.; writing-original draft preparation, G.G.-V. and D.L.-P.; writing-review and editing, G.G.-V., F.Z.-O., D.L.-P., G.B. and J.C.-P.; visualisation, G.G.-V., G.B., W.R.G. and F.Z.-O.; supervision, G.G.-V., G.B., W.R.G. and F.Z.-O. All authors have read and agreed to the published version of the manuscript.

Funding: This research received no external funding.

Conflicts of Interest: The authors declare no conflict of interest. 


\section{References}

1. Baker, L.R.; Cormier, L.A. Disasters and Vulnerable Populations: Evidence-Based Practice for the Helping Professions; Springer: New York, NY, USA, 2014.

2. Leser, K.A.; Looper-Coats, J.; Roszak, A.R. Emergency Preparedness Plans and Perceptions Among a Sample of United States Childcare Providers. Disaster Med. Public Health Prep. 2019, 13, 704-708. [CrossRef]

3. Skowronski, D.M.; Leir, S.; De Serres, G.; Murti, M.; Dickinson, J.A.; Winter, A.-L.; Olsha, R.; Croxen, M.A.; Drews, S.J.; Charest, H.; et al. Children under 10 years of age were more affected by the 2018/19 influenza A(H1N1)pdm09 epidemic in Canada: Possible cohort effect following the 2009 influenza pandemic. Eurosurveillance 2019, 24, 1900104. [CrossRef]

4. Cinesi, C.; Pañuelas, Ó.; Luján, M.; Egea, C.; Masa, J.F.; García, J.; Ferrando, C. Recomendaciones de consenso respecto al soporte respiratorio no invasivo en el paciente adulto con insuficiencia respiratoria aguda secundaria a infección por SARS-CoV-2. Med. Intensiva 2020, 1480, 1-10. [CrossRef]

5. World Health Organization. Coronavirus Disease (COVID-19) Out-Break; WHO: Geneva, Switzerland, 2020.

6. Zhang, L.; Wang, D.C.; Huang, Q.; Wang, X. Significance of clinical phenomes of patients with COVID-19 infection: A learning from 3795 patients in 80 reports. Clin. Transl. Med. 2020, 10, 28-35. [CrossRef] [PubMed]

7. Bahn, G.H. Coronavirus Disease 2019, School Closures, and Children's Mental Health. J. Korean Acad. Child Adolesc. Psychiatry 2020, 31, 74-79. [CrossRef] [PubMed]

8. Szabo, T.G.; Richling, S.; Embry, D.D.; Biglan, A.; Wilson, K.G. From Helpless to Hero: Promoting Values-Based Behavior and Positive Family Interaction in the Midst of COVID-19. Behav. Anal. Pract. 2020, 13, 568-576. [CrossRef]

9. Liang, L.; Ren, H.; Cao, R.; Hu, Y.; Qin, Z.; Li, C.; Mei, S. The Effect of COVID-19 on Youth Mental Health. Psychiatr. Q. 2020, 91, 841-852. [CrossRef]

10. Shigemura, J.; Ursano, R.J.; Morganstein, J.C.; Kurosawa, M.; Benedek, D.M. Public responses to the novel 2019 coronavirus (2019-nCoV) in Japan: Mental health consequences and target populations. Psychiatry Clin. Neurosci. 2020, 74, 281-282. [CrossRef]

11. Cosic, K.; Popovic, S.; Sarlija, M.; Kesedzic, I. Impact of Human Disasters and COVID-19 Pandemic on Mental Health: Potential of Digital Psychiatry. Psychiatr. Danub. 2020, 32, 25-31. [CrossRef]

12. Brown, M.R.G.; Agyapong, V.I.O.; Greenshaw, A.J.; Cribben, I.; Brett-MacLean, P.; Drolet, J.L.; McDonald-Harker, C.; Omeje, J.; Mankowsi, M.; Noble, S.; et al. After the Fort McMurray wildfire there are significant increases in mental health symptoms in grade 7-12 students compared to controls. BMC Psychiatry 2019, 19, 18. [CrossRef]

13. Latham, B.S.; Sullivan, J.; Williams, R.S.; Eakin, M.N. Maintaining Emotional Well-Being During the COVID-19 Pandemic: A Resource for Your Patients. Chronic Obstr. Pulm. Dis. J. COPD Found. 2020, 7, 76-78. [CrossRef]

14. Polizzi, C.; Lynn, S.J.; Perry, A. Stress and coping in the time of covid-19: Pathways to resilience and recovery. Clin. Neuropsychiatry 2020, 17, 59-62. [CrossRef]

15. Casale, S.; Flett, G.L. Interpersonally-based fears during the COVID-19 pandemic: Reflections on the fear of missing out and the fear of not mattering constructs. Clin. Neuropsychiatry 2020, 17, 88-93. [CrossRef]

16. Sadhwani, I. Effect of self-concept on adolescent depression. J. Psychol. Res. 2013, 7, 147-152.

17. Norrington, J. Adolescent Peer Victimization, Self-Concept, and Psychological Distress in Emerging Adulthood. Youth Soc. 2020, 1-23. [CrossRef]

18. Turner, H.A.; Shattuck, A.; Finkelhor, D.; Hamby, S. Effects of Poly-Victimization on Adolescent Social Support, Self-Concept, and Psychological Distress. J. Interpers. Violence 2016, 32, 755-780. [CrossRef] [PubMed]

19. Alonso, D.A.; Mendo-Lázaro, S.; León-Del-Barco, B.; Mirabel-Alviz, M.; Iglesias-Gallego, D. Multidimensional Self-Concept in Elementary Education: Sport Practice and Gender. Sustainability 2018, 10, 2805. [CrossRef]

20. Padial-Ruz, R.; Turpin, J.A.P.; Cepero-González, M.; Zurita-Ortega, F. Effects of Physical Self-Concept, Emotional Isolation, and Family Functioning on Attitudes towards Physical Education in Adolescents: Structural Equation Analysis. Int. J. Environ. Res. Public Health 2019, 17, 94. [CrossRef]

21. Torres, L.H.; Al-Lal, M.; Mohamed, L. Academic Achievement, Self-Concept, Personality and Emotional Intelligence in Primary Education. Analysis by Gender and Cultural Group. Front. Psychol. 2020, 10, 1-13. [CrossRef] 
22. González-Valero, G.; Zurita, F.; Martínez-Martínez, A. Panorama motivacional y de actividad física en estudiantes: Una revisión sistemática. ESHPA Educ. Sport Health Phys. Act. 2018, 1, 41-58. Available online: http://hdl.handle.net/10481/48961 (accessed on 12 June 2020).

23. Chui, W.H.; Wong, M.Y.H. Gender Differences in Happiness and Life Satisfaction Among Adolescents in Hong Kong: Relationships and Self-Concept. Soc. Indic. Res. 2015, 125, 1035-1051. [CrossRef]

24. Onetti-Onetti, W.; Chinchilla-Minguet, J.L.; Martins, F.M.L.; Castillo-Rodríguez, A. Self-Concept and Physical Activity: Differences Between High School and University Students in Spain and Portugal. Front. Psychol. 2019, 10, 1333. [CrossRef] [PubMed]

25. Onyeaka, H.K.; Zahid, S.; Patel, R.S. The Unaddressed Behavioral Health Aspect During the Coronavirus Pandemic. Cureus 2020, 12, e7351. [CrossRef] [PubMed]

26. Akgül, S.; Hüsnü, Ş.; Derman, O.; Özmert, E.; Bideci, A.; Hasanoğlu, E. Mental health of Syrian refugee adolescents: How far have we come? Turk. J. Pediatrics 2019, 61, 839-845. [CrossRef]

27. Karatzias, T.; Murphy, P.; Cloitre, M.; Bisson, J.; Roberts, N.; Shevlin, M.; Hyland, P.; Maercker, A.; Ben-Ezra, M.; Coventry, P.; et al. Psychological interventions for ICD-11 complex PTSD symptoms: Systematic review and meta-analysis. Psychol. Med. 2019, 49, 1761-1775. [CrossRef]

28. Moreno-Alcazar, A.; Treen, D.; Valiente-Gómez, A.; Sio-Eroles, A.; Pérez, V.; Amann, B.L.; Radua, J. Efficacy of Eye Movement Desensitization and Reprocessing in Children and Adolescent with Post-traumatic Stress Disorder: A Meta-Analysis of Randomized Controlled Trials. Front. Psychol. 2017, 8, 1750. [CrossRef]

29. Villalta, L.; Khadr, S.; Chua, K.-C.; Kramer, T.; Clarke, V.; Viner, R.M.; Stringaris, A.; Smith, P. Complex post-traumatic stress symptoms in female adolescents: The role of emotion dysregulation in impairment and trauma exposure after an acute sexual assault. Eur. J. Psychotraumatol. 2020, 11, 1710400. [CrossRef]

30. Orrù, G.; Ciacchini, R.; Gemignani, A.; Conversano, C. Psychological intervention measures during the COVID-19 pandemic. Clin. Neuropsychiatry 2020, 17, 76-79. [CrossRef]

31. Xiang, Y.-T.; Yang, Y.; Li, W.; Zhang, L.; Zhang, Q.; Cheung, T.; Ng, C.H. Timely mental health care for the 2019 novel coronavirus outbreak is urgently needed. Lancet Psychiatry 2020, 7, 228-229. [CrossRef]

32. Castro-Sánchez, M.; Zurita-Ortega, F.; Martínez-Martínez, A.; Chacón-Cuberos, R.; Espejo-Garcés, T. Motivational climate of adolescents and their relationship to gender, physical activity, sport, federated sport and physical activity family. RICYDE Rev. Int. Cienc. Deporte. 2016, 12, 262-277. [CrossRef]

33. Shavelson, J.; Hubner, J.J.; Stanton, G.C. Self-concept: Validation of construct interepretations. Rev. Educ. Res. 1976, 46, 407-442. [CrossRef]

34. García, F.; Musitu, G. AF5: Self-Concept Form 5; TEA Ediciones: Madrid, Spain, 1999.

35. Cohen, J. Statistical Power Analysis for the Behavioral Sciences, 2nd ed.; Erlbaum: Hillsdale, NJ, USA, 1988.

36. Cohen, J. A power primer. Psychol. Bull. 1992, 112, 155-159. [CrossRef] [PubMed]

37. Fraguela-Vale, R.; Varela-Garrote, L.; Carretero-García, M.; Peralbo-Rubio, E.M. Basic Psychological Needs, Physical Self-Concept, and Physical Activity Among Adolescents: Autonomy in Focus. Front. Psychol. 2020, 11, 491. [CrossRef] [PubMed]

38. Garn, A.C.; Morin, A.J.S.; White, R.L.; Owen, K.B.; Donley, W.; Lonsdale, C. Moderate-to-vigorous physical activity as a predictor of changes in physical self-concept in adolescents. Health Psychol. 2020, 39, 190-198. [CrossRef] [PubMed]

39. Ramírez-Granizo, I.A.; Sánchez-Zafra, M.; Zurita-Ortega, F.; Puertas-Molero, P.; González-Valero, G.; Ubago-Jiménez, J.L. Multidimensional Self-Concept Depending on Levels of Resilience and the Motivational Climate Directed towards Sport in Schoolchildren. Int. J. Environ. Res. Public Health 2020, 17, 534. [CrossRef] [PubMed]

40. Povedano-Diaz, A.; Povedano-Díaz, A.; Vera, M. Adolescents' Life Satisfaction: The Role of Classroom, Family, Self-Concept and Gender. Int. J. Environ. Res. Public Health 2019, 17, 19. [CrossRef] [PubMed]

41. Zubeldia, M.; Diaz, M.; Goñi, E. Self-concept, attributions and trait-anxiety in conservatory students. Differences related to age and gender. Psychol. Soc. Educ. 2018, 10, 79-102. [CrossRef]

42. Ramos-Díaz, E.; Rodríguez-Fernández, A.; Antonio-Agirre, I. Self-concept and subjective well-being being based on gender and educational lever in adolescence. Psicol. Educ. 2017, 23, 89-94. [CrossRef]

43. Park, S.; Yun, D.-H.; Kwon, I.-S. Relationships of Adolescents' Physical Self-Concept and Life Satisfaction by Gender and Physical Activity Level. Korean J. Sport Stud. 2018, 57, 195-205. [CrossRef]

44. Martínez-Marín, M.D.; Martínez, C.; Paterna, C. Gendered self-concept and gender as predictors of emotional intelligence: A comparison through of age. Curr. Psychol. 2020, 1-14. [CrossRef] 
45. Suriá-Martínez, R.; Ortigosa-Quiles, J.M.; Riquelme-Marin, A. Emotional Intelligence Profiles of University Students with Motor Disabilities: Differential Analysis of Self-Concept Dimensions. Int. J. Environ. Res. Public Health 2019, 16, 4073. [CrossRef] [PubMed]

46. Parise, M.; Canzi, E.; Olivari, M.G.; Ferrari, L. Self-concept clarity and psychological adjustment in adolescence: The mediating role of emotion regulation. Pers. Individ. Differ. 2019, 138, 363-365. [CrossRef]

47. Cachón-Zagalaz, J.; Sanabrias-Moreno, D.; Sánchez-Zafra, M.; Lara-Sánchez, A.J.; Lara-Sánchez, A.J. Use of the Smartphone and Self-Concept in University Students According to the Gender Variable. Int. J. Environ. Res. Public Health 2020, 17, 4184. [CrossRef] [PubMed]

48. Kulakow, S. Academic self-concept and achievement motivation among adolescent students in different learning environments: Does competence-support matter? Learn. Motiv. 2020, 70, 101632. [CrossRef]

49. Galindo-Domínguez, H. Standardization of the self-concept AF-5 scale by school year and gender in primary education. Psicol. Educ. 2019, 25, 117-125. [CrossRef]

50. Meza-Pena, C.; Pompa-Guajardo, E.G. Gender, obesity and self concept in a sample of Mexican adolescents. RICYDE Rev. Int. Cien. Dep. 2016, 12, 137-148. [CrossRef]

51. Tapia-López, A. Gender differences in physical activity levels, degree of adherence to the Mediterranean diet, and physical self-concept in adolescents. Retos 2019, 36, 185-192.

52. Xie, F.; Xin, Z.; Chen, X.; Zhang, L. Gender Difference of Chinese High School Students' Math Anxiety: The Effects of Self-Esteem, Test Anxiety and General Anxiety. Sex Roles 2018, 81, 235-244. [CrossRef]

53. Brown, D.M.; Cairney, J. The synergistic effect of poor motor coordination, gender and age on self-concept in children: A longitudinal analysis. Res. Dev. Disabil. 2020, 98, 103576. [CrossRef]

54. Chalabaev, A.; Sarrazinr, P.; Fontayne, P.; Boiché, J.; Clément-Guillotin, C. The influence of sex stereotypes and gender roles on participation and performance in sport and exercise: Review and future directions. Psychol. Sport Exerc. 2013, 14, 136-144. [CrossRef]

55. Clevinger, K.; Petrie, T.; Martin, S.; Greenleaf, C. The Relationship of Sport Involvement and Gender to Physical Fitness, Self-Efficacy, and Self-Concept in Middle School Students. Phys. Educ. 2020, 77, 154-172. [CrossRef]

56. Gómez-Fernández, N.; Albert, J.-F. Physical activity in and out-of-school and academic performance in Spain. Health Educ. J. 2020, 79, 1-15. [CrossRef]

57. Masoomi, H.; Taheri, M.; Irandoust, K.; H'Mida, C.; Chtourou, H. The relationship of breakfast and snack foods with cognitive and academic performance and physical activity levels of adolescent students. Biol. Rhythm. Res. 2019, 51, 481-488. [CrossRef]

58. Ishii, K.; Aoyagi, K.; Shibata, A.; Koohsari, M.J.; Carver, A.; Oka, K. Joint Associations of Leisure Screen Time and Physical Activity with Academic Performance in a Sample of Japanese Children. Int. J. Environ. Res. Public Health 2020, 17, 757. [CrossRef]

59. Carriedo, A.; González, C. Academic Achievement in Physical Education: Academic versus Physical Activity aspects. Cult. Cienc. Deporte 2019, 14, 225-232. [CrossRef]

60. Louise, T.; Hernández, A.; Reigal, R.E.; Morales, V. Effects of physical activity on self-concept and self-efficacy in preadolescents. Retos 2016, 29, 61-65.

61. Grant, P.M.; Perivoliotis, D.; Luther, L.; Bredemeier, K.; Beck, A.T. Rapid improvement in beliefs, mood, and performance following an experimental success experience in an analogue test of recovery-oriented cognitive therapy. Psychol. Med. 2017, 48, 261-268. [CrossRef]

62. Neto, L.D.O.; Elsangedy, H.M.; Tavares, V.D.D.O.; Teixeira, C.V.L.S.; Behm, D.G.; Da Silva-Grigoletto, M.E. TrainingInHome-Home-based training during COVID-19 (SARS-COV2) pandemic: Physical exercise and behavior-based approach. Rev. Bras. Fisiol. Exerc. 2020, 19, 9-19. [CrossRef]

63. Aubertin-Leheudre, M.; Rolland, Y. The Importance of Physical Activity to Care for Frail Older Adults during the COVID-19 Pandemic. J. Am. Med. Dir. Assoc. 2020, 21, 973-976. [CrossRef]

64. Vetrovsky, T.; Frybova, T.; Gant, I.; Semerad, M.; Cimler, R.; Bunc, V.; Siranec, M.; Miklikova, M.; Vesely, J.; Griva, M.; et al. The detrimental effect of COVID-19 nationwide quarantine on accelerometer-assessed physical activity of heart failure patients. ESC Hear. Fail. 2020, 1-5. [CrossRef]

65. Qin, F.; Song, Y.; Nassis, G.P.; Zhao, L.; Cui, S.; Lai, L.; Wu, Z.; Xu, M.; Qu, C.; Dong, Y.; et al. Prevalence of Insufficient Physical Activity, Sedentary Screen Time and Emotional Well-Being During the Early Days of the 2019 Novel Coronavirus (COVID-19) Outbreak in China: A National Cross-Sectional Study. SSRN Electron. J. 2020, 1-28. [CrossRef] 
66. Chen, P.; Mao, L.; Nassis, G.P.; Harmer, P.; Ainsworth, B.E.; Li, F. Coronavirus disease (COVID-19): The need to maintain regular physical activity while taking precautions. J. Sport Health Sci. 2020, 9, 103-104. [CrossRef] [PubMed]

67. Lippi, G.; Henry, B.M.; Sanchis-Gomar, F. Physical inactivity and cardiovascular disease at the time of coronavirus disease 2019 (COVID-19). Eur. J. Prev. Cardiol. 2020, 27, 906-908. [CrossRef] [PubMed]

68. Di Stefano, V.; Battaglia, G.; Giustino, V.; Gagliardo, A.; D’Aleo, M.; Giannini, O.; Palma, A.; Brighina, F. Significant reduction of physical activity in patients with neuromuscular disease during COVID-19 pandemic: The long-term consequences of quarantine. J. Neurol. 2020, 1-7. [CrossRef]

69. Van De Venis, L.; Van De Warrenburg, B.P.C.; Weerdesteyn, V.; Van Lith, B.J.H.; Geurts, A.C.H.; Nonnekes, J. COVID-19 reveals influence of physical activity on symptom severity in hereditary spastic paraplegia. J. Neurol. 2020, 236, 1-3. [CrossRef]

70. Lim, M.A.; Pranata, R. Sports activities during any pandemic lockdown. Ir. J. Med Sci. 2020, 1-5. [CrossRef]

71. Park, S.; Kim, B.; Lee, J. Social Distancing and Outdoor Physical Activity During the COVID-19 Outbreak in South Korea: Implications for Physical Distancing Strategies. Asia Pac. J. Public Health 2020, 32667221, 1-3. [CrossRef]

72. Dwyer, M.J.; Pasini, M.; De Dominicis, S.; Righi, E. Physical activity: Benefits and challenges during the COVID-19 pandemic. Scand. J. Med. Sci. Sports 2020, 30, 1291-1294. [CrossRef]

73. Pieh, C.; Budimir, S.; Probst, T. The effect of age, gender, income, work, and physical activity on mental health during coronavirus disease (COVID-19) lockdown in Austria. J. Psychosom. Res. 2020, 136, 110186. [CrossRef]

74. Shahidi, S.H.; Williams, J.S.; Hassani, F. Physical activity during COVID-19 quarantine. Acta Paediatr. 2020, 1-2. [CrossRef]

75. Blocken, B.; Van Druenen, T.; Van Hooff, T.; Verstappen, P.; Marchal, T.; Marr, L. Can indoor sports centers be allowed to re-open during the COVID-19 pandemic based on a certificate of equivalence? Build. Environ. 2020, 180, 107022. [CrossRef] [PubMed]

76. Buldú, J.; Antequera, D.R.; Aguirre, J. The resumption of sports competitions after COVID-19 lockdown: The case of the Spanish football league. Chaos Solitons Fractals 2020, 138, 109964. [CrossRef] [PubMed]

77. Stokes, K.A.; Jones, B.; Bennett, M.; Close, G.L.; Gill, N.; Hull, J.H.; Kasper, A.M.; Kemp, S.P.; Mellalieu, S.D.; Peirce, N.; et al. Returning to Play after Prolonged Training Restrictions in Professional Collision Sports. Int. J. Sports Med. 2020, 2-3. [CrossRef] [PubMed]

78. Matias, T.S.; Dominski, F.H.; Marks, D. Human needs in COVID-19 isolation. J. Health Psychol. 2020, 25, 871-882. [CrossRef] [PubMed]

79. World Health Organization (WHO). Global Recommendations on Physical Activity for Health; WHO Press: Geneva, Switzerland, 2010; pp. 15-21.

80. Miotto, K.; Sanford, J.; Brymer, M.J.; Bursch, B.; Pynoos, R.S. Implementing an emotional support and mental health response plan for healthcare workers during the COVID-19 pandemic. Psychol. Trauma Theory Res. Pract. Policy 2020, 12, S165-S167. [CrossRef]

81. Ramos-Díaz, E.; Axpe, I.; Fernández-Lasarte, O.; Jiménez-Jiménez, V. Cognitive-behavioral intervention to enhance self-concept in a case of an adolescent victim of emotional maltreatment. Rev. Clin. Contemp. 2018, 9,1-10. [CrossRef]

(C) 2020 by the authors. Licensee MDPI, Basel, Switzerland. This article is an open access article distributed under the terms and conditions of the Creative Commons Attribution (CC BY) license (http://creativecommons.org/licenses/by/4.0/). 